(Aus dem Institut für allg. und exper. Pathologie der kgl. ung. Universität Budapest. Direktor: Professor Dr. Franz Tangl.)

\title{
Untersuchungen über die Wärmetönung von Enzymreaktionen.
}

\author{
IV. Mitteilung. \\ Über die Wärmetönnng der Pepsinverdanung des Eiweisses. \\ Von
}

Dr. Paul Hári,

Privatdozent und I. Assistent des Institutes.

Die Ergebnisse einiger Versuche, die R. v. Lengyel zur Klärung der Frage nach der Wärmetönung des Pepsinverdauung anstellte, gestatteten ihm, in einer kurzen vorläufigen Mitteilung ${ }^{1}$ ) mit ziemlicher Wahrscheinlichkeit auszusprechen, dass die Reaktionswärme der Pepsinverdaunng des Eiweisses gleich Null oder doch jedenfalls äusserst gering sei.

Da diese Versuche jedoch keine definitive Lösung der Frage ergaben, schien es mir geboten, ihr auf Grund der Erfahrungen, die ich bei der Prüfung der Reaktionswärme der Trypsinverdauung ${ }^{2}$ ) gesammelt habe, näher zu treten.

Das Prinzip der Versuchsanordnung war dasselbe wie. in Lengyel's Arbeit sowie in meiner Trypsinarbeit: Ein Verdauungsgemisch von bekanntem Inhalte an chemischer Energie wurde nach einer angemessenen Verdauungsperiode im ganzen eingedampit und der Gehalt des eingetrockneten Rückstandes an chemischer Energie bestimmt. Ein Vergleich des Energiegehaltes vor und nach der Verdauung muss dann entweder ergeben, dass der Energiegehalt während der Verdauung zu- oder abgenommen hat oder aber, dass er unverändert geblieben ist.

1) R. v. Lengyel, Einige Versuche über die Wärmetönung der Pepsinverdauung des Eiweisses. Pflüger's Arch. Bd. 115 S. 7.

2) Pau.l Hári, Über die Wärmetönung der Trypsinverdauung des Eiweisses. PfIüger's Arch. Bd. 115 S. 11. 
Auch die Einzelheiten der Versuchsanordnung waren dieselben wie bei der Trypsinarbeit, daher bezüglich aller Details - die sich auf die Bestimmung des Gehaltes an Trockensubstanz, Asche, Stickstoff, chemischer Energie, ferner auf das Ansetzen und das Eindampfen der Verdauungsgemische usw. beziehen - auf die erwähnte Trypsinarbeit verwiesen sei.

\section{I.}

Es sollen zunächst die Ergebnisse zweier Versuchsreihen mitgeteilt werden, von denen die eine in der Zusammensetzung und Konzentration des Verdauungsgemisches der Lengyel'sehen Versuchsanordnung entsprach, die andere aber nur in Anwendung einer stärker konzentrierten Säure von ersterer sich unterschied.

\section{Versuchsreihe.}

Merck'sches Ovalbumin wurde mit fein gepulvertem Pepsinum purum in lamellis von Merek und kristallisierter Oxalsäure im Verhältnis von $89,4: 1,8: 8,8$ vermischt und von diesem Gemische je ca. $3 \mathrm{~g}$ mit $100 \mathrm{~g}$ destillierten Wassers und reichlich Toluol angesetzt.

Das Pulvergemisch enthielt:

\begin{tabular}{|c|c|c|c|}
\hline & lufttrocken & $\begin{array}{c}\text { in der Trocken- } \\
\text { substanz }\end{array}$ & $\begin{array}{l}\text { in der aschenfreien } \\
\text { Trockensubstanz }\end{array}$ \\
\hline Wasser & $12,40 \%$ & - & - \\
\hline... & $5,44 \%$ & $6,21 \%$ & - \\
\hline & $11,69 \%$ & $13,34 \%$ & $14,22 \%$ \\
\hline Spezifischer Energiegehalt & 4506 cal. & 5144 cal. & 5484 cal. \\
\hline
\end{tabular}

Die saure Lösung enthielt ca. $0,2 \%$ wasserfreie Oxalsäure.

In den frisch angesetzten Gemischen waren $31,6 \%$ des $\mathrm{N}$ in Form von koagulablem Eiweiss vorhanden.

Die Eindampfung der sofort verarbeiteten sowie auch der vorher verdauten Portionen erfolgte ohne Abstumpfen der Säure.

\section{Versuchsreihe.}

Zusammenstellung des Pulvers wie in der ersten Versuchsreihe, nur in abweichendem Verbältnis von Eiweiss : Pepsin: Oxalsäure gleich $81,8: 1,8: 16,4$.

Das Ansetzen der Gemische und deren Aufarbeiten erfolgten wie in der 1 . Versuchsreihe.

Das Pulvergemisch enthielt: 
Untersuchungen über die Wärmetönung von Enzymreaktionen. IV. 461

\begin{tabular}{|c|c|c|c|}
\hline & lufttrocken & $\begin{array}{c}\text { in der Trocken- } \\
\text { substanz }\end{array}$ & $\begin{array}{l}\text { in der aschenfreien } \\
\text { Trockensubstanz }\end{array}$ \\
\hline Wasser ....... & $13,40 \%$ & - & - \\
\hline Asche . . . & $4,99 \%$ & $5,76 \%$ & - \\
\hline & $11,05 \%$ & $12,76 \%$ & $13,54 \%$ \\
\hline Spezifischer Energiegehalt & 4257 cal. & 4916 cal. & 5216 cal. \\
\hline
\end{tabular}

Die Konzentrationen der wasserfreien Oxalsäure betrug in der angesetzten Lösung ca. $0,4 \%$.

Wie aus den Daten der Tab. I ersichtlich, verursacht die einfache Eindampfung obne vorangehende Verdaung (Versuch 1 und 2) eine nicht unerhebliche Zunahme der Trockensubstanz $(0,60-1,14 \%)$ und eine geringe Abnahme des Energiegehaltes; in den verdauten Gemischen dagegen ist eine bedeutendere Zunahme der Trockensubstanz und auch eine Zunahme des Energiegehaltes zu konstatieren, die keine Proportionalität mit der Dauer der Verdauung aufweist.

Es fragt sich nun, ob man 'aus dieser Versuchsreihe bereits den Schluss ziehen kann, dass die Wärmetönung der Pepsinverdauung eine negative ist. Denn die ausgesprochene Zunahme des Energiegehaltes - auf die es hier doch hauptsächlich ankommt - kann entweder

1. in der Tat von der Pepsinverdauung selbst herrübren oder aber

2. von chemischen Prozessen anderer Art, die in der eigentümlichen Versuchsanordnung begründet sind und speziell thermisch vielleicht nicht neutral verlaufen. Und zwar kann

a) während der ganzen Dauer der sogenannten Verdauung neben der Pepsinverdauung eine einfache Säurewirkung (im Sinne der Bildung von Säurealbuminat) vor sich gehen, ja vielleicht jene an Intensität übertreffen, oder aber ist

b) die Zunabme des Energiegehaltes von der Säurewirkung bedingt, die während des Eindampfens der Gemische stattfindet, indem ja die Oxalsäure in dieser Versuchsreihe (wie auch in Lengyel's Versuchen) vorher nicht abgestumpft ward, zudem während des Eindampfens an Konzentration immerfort noch zugenommen hatte.

Gegen die Annahme, dass hier eine richtige Pepsinverdaung vor sich gegangen wäre, spricht der Umstand, dass die Menge des koagulablen Eiweisses auffallend langsam abgenommen hat. Um nämlich einen Maassstab für das Fortschreiten der Verdauung zu 


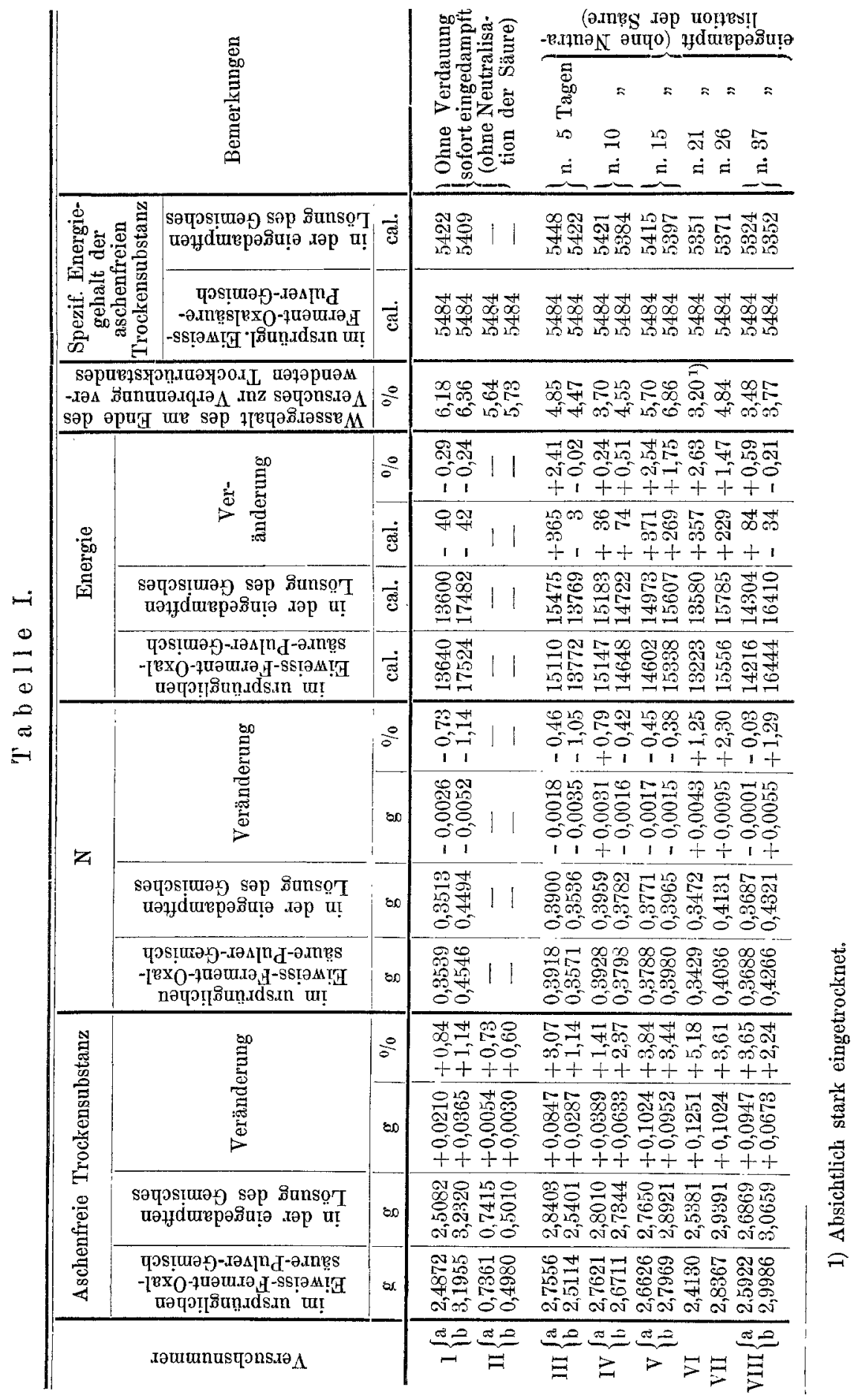


haben, wurde jedesmal nach Ablauf einer bestimmten Verdauungsperiode in einem der angesetzten Gemische die Menge des noch koagulablen Eiweisses resp. $\mathrm{N}$ bestimmt.

Aus nachstehender Zusammenstellung ist ersichtlich, dass vom ursprünglich koagulabel gewesenen Eiweiss nach 5 Tagen noch ca. $75 \%$, nach 37 Tagen aber immer noch ca. 10\% koagulabel waren. Die Menge des koagulablen $\mathrm{N}$ betrug:

$$
\begin{aligned}
& \text { Vor der Verdauung. . . . . 31,6\% } \\
& \text { Nach } 5 \text { Tagen . . . . . . 23,2\% } \\
& " 10 \quad \text { " } 10.0 . \quad . \quad .18,7 \% \\
& " 15 \quad \text { n } \quad . \quad . \quad . \quad . \quad . \quad 6,2 \% \\
& \text { " } 26 \quad \text {. . . . . . } 5,7 \% \\
& \text { " } 37 \% \quad . \quad . \quad . \quad . \quad . \quad . \quad 3,3 \%
\end{aligned}
$$

Von einer einigermaassen kräftigen Pepsinverdauung - wie sie 7. B. in der 5. Verauchsreihe (S. 468) beschrieben werden wird, wo nach 2 Tagen bereits keine Spur von koagulablem Eiweiss vorhanden war, kann in dieser 1. Versuchsreihe sicher nicht die Rede sein, daher eher anzunebmen war, dass die Zunahme der Trockensubstanz und des Energiegehaltes von den weiter oben sub a) und b) erwähnten Säurewirkungen herrühren. - Ist diese Vermutung richtig, so musste in einer 2. Versuchsreihe, in der die Konzentration der Oxalsäure das Doppelte der 1. Versuchsreihe betrug, die stärkere Säurewirkung während des Eindampfens (b) an einer stärkeren Zunahme der Trockensubstanz und des Energiegehaltes der unverdauten Gemische zu erkennen sein, die stärkere Säurewirkung während der sogenannten Verdauung aber (a) an einer weiteren inteusiven Zunahme der Trockensubstanz und des Energiegehaltes der verdauten Gemische.

Wie ein Blick auf Tab. II uns lehrt, traf obige Annahme bezüglich der Trockensubstanz vollinhaltlich und im Sinne beider Säurewirkungen ein; denn

a) das Eindampfen mit der stärkeren Säure hatte zur Folge, dass die Zunahme der Trockensubstanz der ohne Verdauung eingedampften Gemische 3,42-3,52\% betrug, das ist mehr als das Dreifache der entsprechenden Zunahme in der 1. Versuchsreihe;

b) an den verdauten Gemischen war gar - neben einer unverkennbaren starken Progression mit zunehmender Dauer der Einwirkung - eine Zunahme bis zu 9,37 resp. 12,11\% zu konstatieren, 
während der höchste auf diese Art erreichte Wert in der 1. Versuchsreihe bloss $3,84 \%$ betrug.

Wenn wir noch in Betracht ziehen, dass die Abnahme des koagulablen $\mathrm{N}$ in dieser Versuchsreihe zwar rascher als in der ersten erfolgte, vom koaguablen $\mathrm{N}$ aber nach 4 Tagen immer noch ca. $30 \%$, nach 10 Tagen aber ca. 20\% koagulabel blieben, so dass von einer kräftigen Pepsinverdauung auch in dieser Versuchsreihe nicht die Rede sein konnte, können wir aussagen, dass die in diesen beiden Versuchsreihen konstatierbare Zunahme der Trockensubstanz nicht als Wirkung einer Pepsinverdaung, sondern hauptsächlich - wenn nicht ausschliesslich — als säurewirkung aufzufassen is t.

Je klarer es nun ist, dass die Zunahme der Trockensubstanz auf einer Säurewirkung beruht, um so merkwürdiger erscheint das Verhalten des Energiegehaltes. Während nämlich in den obne Verdauung eingedampften Gemischen dieser 2. Versuchsreihe ein $0,70-0,88 \%$ betragender Energieverlust (gegen $0,24-0,29 \%$ in der 1. Versuchsreihe) zu konstatieren war, weisen die vorher verdauten Gemische zwar eine Zunahme des Energiegehaltes auf, diese zeugte aber bloss eine Abhängigkeit vom Wassergehalt des eingetrockneten Rückstandes, d. h. sie fiel im grossen und ganzen um so geringer aus, je stärker der Rüekstand eingetrocknet war; sie wies jedoch keine Progression mit zunehmender Dauer der Verdaung auf, und insbesondere war sie nicht wesebtlich grösser als in den verdauten Gemischen der 1. Versuchsreihe mit der schwächeren Säure. - Andererseits musste es aber bereits in der 1. Versuchsreihe auffallen, dass der Energiezuwachs der am längsten verdauten Gemische (Versuch VIII a und VIIIb) den kürzer verdauten gegenüber einen ansehnlichen Abfall aufweist, und wenn auch zufälligerweise die Gemische gerade in diesen Versuchen am stärksten eingetrocknet wurden (wie aus dem geringen Wassergebalt des Eintrocknungsrückstandes zu ersehen ist), der Abfall des Energiezuwachses also hierin seine Erklärung finden möchte, konnte ich die Möglichkeit einer anderen Erklärung doch nicht von der Hand weisen. Ich dachte an die Möglichkeit, dass Säurewirkung und Pepsinverdauung - jene mit einer negativen, diese mit einer positiven Wärmetönung - konkurrierende Prozesse sind, deren Resultierende in obigen Versuchen - wo die Säure 
evident stark, das Pepsin aber evident schwach wirkten - - darin bestand, dass den ersten Stadjen des sogenannten Verdauungsprozesses zwar die Säurewirkung ihren Stempel aufdrückte, später aber die Pepsinverdauung sich geltendmacht, und zwar im Sinne eines Abfalles des Energiezuwachses.

Um hierüber Klarheit zu erhalten, musste ich nun ein durch verhältnismässig kurze Zeit verdautes Gemisch absichtlich sehr stark, ein anderes verhältnismässig lang verdautes Gemisch vorsichtig, d. b. so eindampfen, dass es möglichst wenig eintrockne. Ist nämlich der Abfall der Energiezunahme bloss eine Funktion des Eintrocknungsgrades, so musste ich im ersteren Gemisch einen sehr starken oder gar unter allen Gemischen den stärksten Energieabfall erhalten, im anderen wenig eingetrockneten Gemische aber nicht nur keinen Abfall des Energiezuwachses erhalten, sondern im Gegenteil den stärksten Energiezuwachs unter allen Versuchen konstatieren; war hingegen meine Annahme richtig, hängt also der Abfall des Energiezuwachses (ausser vom Eintrocknungsgrade) auch noch von der längeren Dauer der Verdauung ab, so musste die Zunahme des Energiegehaltes im ersteren Falle trotz der sehr starken Eintrocknung bedeutencier sein als in den nachfolgenden Versuchen mit der längeren Verdauungsdauer, in den anderen Gemischen aber, trotz der schonenden Eintrocknung, am geringsten unter allen Versuchen ausfallen.

Wie aus Tab. II zu ersehen ist, haben die Tatsachen meinen Erwartungen nicht vollinhaltlich, wenigstens nicht mit hinlänglicher Deutlichkeit, entsprochen :

Im VI. Versuch der 1. Versuchsreihe war allerdings der Energiezuwachs, wie ich erwartet habe, trotz der starken Eintrocknung grösser als in den weiteren Versuchen. Auch zeigten die Gemische IV a und IV b trotz der schonenden Eintrocknung einen deutlichen Abfall gegen die vier tagelang verdauten Gemische in den VerVersuchen II a und II b, was ebenfalls meiner Erwartung entsprach; der Energiezuwachs dieser Gemische war aber grösser als in den kürzer verdauten der Versuche III a und III $b$, die allerdings abnorm stark eingetrocknet wurden.

Das Verhalten des Gemisches VI der 1. Versuchsreihe sprach deutlich für, das der Gemische IV a und IVb weder für noch gegen meine Annahme zweier thermisch entgegengesetzt gearteter Prozesse, deren Resultierende schliesslich vom Überwiegen des einen oder des anderen abhinge. 





II.

Da jch mir von weiteren Versuchen ähnlicher Anordnung keine befriedigende Lösung der obschwebenden Frage versprechen konnte, musste ich daran gehen, Säurewirkung und Pepsinwirkung, jede für sich, gesondert $z u$ untersuchen.

Über die reine Säurewirkung konnte ich Aufschluss nur von solchen Versuchen erwarten, in denen die Säure ihre Wirksamkeit nur während der sogenannten Verdauung ausüben konnte - jedoch nicht während des Eindampfens -, was durch sorgfältiges Neutralisieren vor dem Eindampfen verhütet werden musste. Da es aber möglich war, dass auch das Eindampfen mit der neutralisierten Säure, d. h. mit einer verdünnten Salzlösung, ja sogar mit destilliertem Wasser allein schon Veränderungen in der Trockensubstanz und im Energiegehalt der eingedampften Gemische verursacht, mussten auch diese Fragen durch eigene Versuchsreihen geklärt werden.

Die Pepsinverdaung musste ihrerseits aus vergleichenden Untersuchungen solcher Verdauungsgemische hervorgehen, deren eine bloss mit Säure, die anderen aber mit Säure und Pepsin angesetzt, sonst aber unter ganz identischen Bedingungen verarbeitet wurden.

Die folgenden Versuchsreihen 3, 4 und 5 dienten diesen Untersuchungen.

\section{Versuchsreihe.}

Merck'sches Ovalbumin wurde mit destilliertem Wasser (einmal oder wiederholt) eingedampft. Da die Versuche $\mathbf{z u}$ verschiedenen Zeiten vorgenommen wurden und das verwendete Eiweisspulver als verschieden feucht sich erwies, sollen hier nur die auf Trockensubstanz und aschenfreie Trockensubstanz berechneten Daten angeführt sein. Das Eiweisspulver enthielt:

\begin{tabular}{|c|c|c|}
\hline & $\begin{array}{l}\text { in der Trocken- } \\
\text { substanz }\end{array}$ & $\begin{array}{l}\text { in der aschenfreien } \\
\text { Troekensubstanz }\end{array}$ \\
\hline & . $6,66 \%$ & - \\
\hline scher Energiegehalt & 5362 cal. & 5745 cal. \\
\hline
\end{tabular}

4. Versuchsreihe.

Von Merck'schem Ovalbumin wurden je ca. $4 \mathrm{~g}$ mit $175 \mathrm{ccm}$ destillierten Wassers und $25 \mathrm{ccm}{ }^{\mathrm{n} / 1}$-Salzsäure und reichlich Toluol versetzt; sodann wurden einige Portionen sofort verarbeitet, andere verschieden lang im Thermostaten belassen und nachher erst eingedampft. Vor dem Eindampfen wurde die Säure in den verdauten 
Gemischen sowohl wie in den nichtverdauten immer erst durch $\mathrm{Zu}$ -



Die saure Lösung enthielt Salzsäure in einer Konzentration von 0,46\%. - Das Pulver selbst hatte folgende Zusammensetzung:

$\begin{array}{lccc} & \text { lnfttrocken } & \begin{array}{c}\text { in der Trocken. } \\ \text { substanz }\end{array} & \begin{array}{c}\text { in der aschenfreien } \\ \text { Trockensubstanz }\end{array} \\ \text { Wasser . . . . . } & 6,94 \% & - & - \\ \text { Asche. . . . . . } & 6,20 \% & 6,66 \% & - \\ \text { N . . . . . . } & 12,64 \% & 13,58 \% & 14,55 \% \\ \text { Spezif. Energiegehalt } & 4993 \text { cal. } & 5365 \mathrm{cal} . & 5748 \mathrm{cal} .\end{array}$

\section{Versuchsreihe.}

M e r ck'sches Ovalbumin wurde mit fein pulverisiertem Pepsinum purum in lamellis Merck im Verbältnis von 90:10 vermischt und von diesem Pulver je ca. $4 \mathrm{~g}$ mit $175 \mathrm{cem}$ destillierten Wassers und $25 \mathrm{ccm}$ n/1-Salzsäure und reichlich Toluol versetzt. Verfahren im übrigen genau wie in der 2. Versuchsreihe.

Das Pulvergemisch enthielt:

\begin{tabular}{|c|c|c|c|}
\hline & Iufttrocken & $\begin{array}{l}\text { in der Trocken- } \\
\text { substanz }\end{array}$ & $\begin{array}{l}\text { in der aschenfreien } \\
\text { Trockensubstanz }\end{array}$ \\
\hline Wasser .... & $9,08 \%$ & - & - \\
\hline Asche. . & $5,60 \%$ & $6,15 \%$ & - \\
\hline $\mathrm{N} \ldots \ldots$ & $12,38 \%$ & $13,61 \%$ & $14,51 \%$ \\
\hline $\mathrm{C} \ldots \ldots$ & - & $52,37 \%$ & $55,80 \%$ \\
\hline $\mathrm{H} \ldots \ldots$ & - & $7,03 \%$ & $7,49 \%$ \\
\hline Spezif. Energiegehalt & 4849 cal. & 5333 cal. & $5683 \mathrm{cal}$. \\
\hline
\end{tabular}

\section{A. Eindampfen mit destilliertem Wasser.}

Die in der Tabelle III angeführten Daten zeigen, dass das Eiweisspulver in destilliertem Wasser gelöst und dann eingetrocknet eine nicht unerhebliche Veränderung in seiner Trockensubstanz und in seinem Gehalt an chemischer Energie erfährt.

Was zunächst die Trockensubstanz anbelangt, sehen wir, dass die einmalige Eindampfung einen Verlust an Trockengehalt verursacht,

1) Die aus dem eingedampften Rückstand gepressten Pastillen enthielten (ausser der ursprünglichen Asche des Eiweisspulvers) bis $25 \%$ Kochsalz, das aus dem Zusatz von Salzsäure und Natronlauge entstand, verbrannten aber in der kalorimetrischen Bombe trotzdem glatt und hinterliessen in den meisten Fällen gar keine, zuweilen geringe Mengen unverbrannter Kohle. Diese wurden in fünf Versuchen durch Wägung bestimmt, und zwar fand ich:
1. $0,0001 \mathrm{~g}$.
2. $0,0003 \mathrm{~g}$.
3. $0,0001 \mathrm{~g}$.
4. ิ $\mathrm{g}$,
5. 의. 
Untersuchungen über die Wärmetönung ron Enzymreaktionen. IV. 469

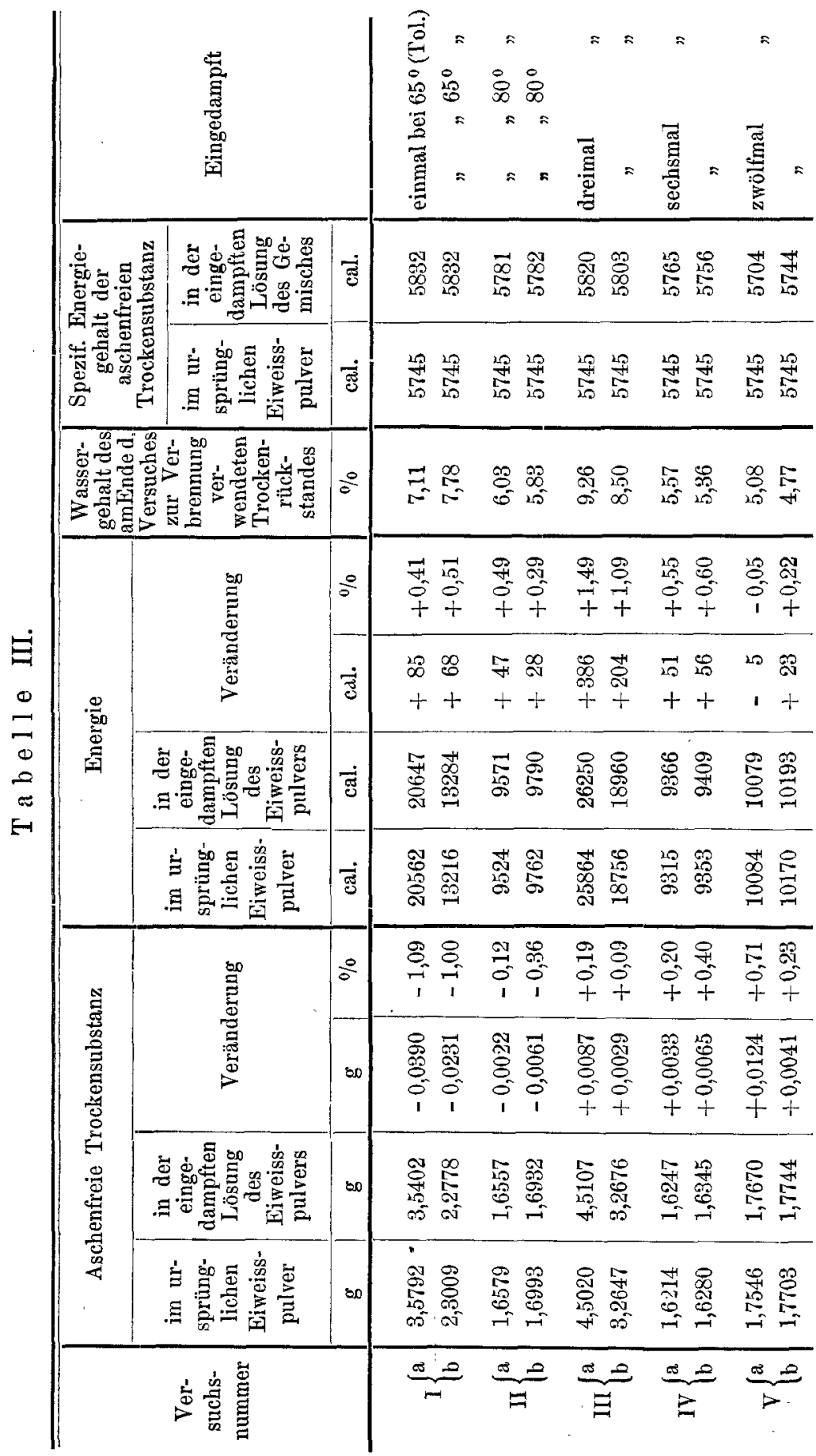


der einmal (als das Eindampfen bei $65^{\circ}$ C. 24 Stunden gedauert hatte) 1,09 resp. 1,00\% betrug, ein anderes Mal (als das Eindampfen der geringeren Menge bei $80^{\circ} \mathrm{C}$. schon in 5 Stunden vollendet war) bloss 0,12 resp. $0,36 \%$ ausmachte.

Wenn der eingetrocknete Rückstand wieder mit destilliertem Wasser angesetzt und eingetrocknet wurde, war an Stelle eines Verlustes an Trockengehalt bereits eine Zunahme desselben getreten, und zwar bei dreimaliger Wiederholung des Eindampfens von 0,19 resp. $0,09 \%$, bei sechsmaliger Wiederholung von 0,20 resp. $0,40 \%$ und bei zwölfmaliger Wiederholung von 0,71 resp. $0,23 \%$. Die Mittelwerte aus je zwei Parallelversuchen stellen folgende Reihe dar:

$$
\begin{aligned}
& \text { einmal . . . . }-1,04 \% \\
& \text { einmal . . . . }-0,24 \% \\
& \text { dreimal . . . . }+0,14 \% \\
& \text { secbsmal . . . + }+0,30 \% \\
& \text { zwölfmal . . . }+0,47 \% \text {. }
\end{aligned}
$$

Dieser Übergang vom anfänglichen Gewichtsverlust zu steigender Gewichtsvermehrung ist nicht anders zu erklären, als dass hier gleichzeitig zwei Prozesse vor sich gehen: eine Verringerung des Trockengehaltes durch das Entweichen flühtiger Zersetzungsprodukte und eine Zunahme derselben durch intramolekulare Wasseraufnahme. Die schliesslich resultierende Veränderung im Gewicht der Trockensubstanz wird davon abhängen, welcher von den beiden Prozessen die Überhand gewinnt. Bei wiederholter Eindampfung war in meinen Versuchen stets eine Zunahme infolge überwiegender Wasseraufnahme zu konstatieren.

Dass die Verringerung der Trockensubstanz durch das Ent-

\begin{tabular}{|c|c|c|c|}
\hline $\begin{array}{c}\text { Veränderung } \\
\text { des }\end{array}$ & $\begin{array}{l}\text { Wassergehalt des } \\
\text { eingetrockneten }\end{array}$ & $\begin{array}{c}\text { Veränderung } \\
\text { des }\end{array}$ & $\begin{array}{l}\text { Wassergebalt des } \\
\text { eingetrockneten }\end{array}$ \\
\hline $\begin{array}{c}\text { Trockengehaltes } \\
-1,09 \% \\
-1,00 \%\end{array}$ & $\left.\begin{array}{c}\text { Rückstandes } \\
\mathbf{7 , 1 1} \% \\
\mathbf{7 , 7 8} \%\end{array}\right\}$ & $\begin{array}{l}\text { Trockengehaltes } \\
\qquad \begin{array}{l}(+0,20 \%) \\
(+0,40 \%)\end{array}\end{array}$ & $\left.\begin{array}{c}\text { Rückstandes } \\
(5,57 \%) \\
(5,36 \%)\end{array}\right\}$ \\
\hline$-0,12 \%$ & $6,03 \%$ & $+0,71 \%$ & $5,08 \%$ \\
\hline$-0,36 \%$ & $5,83 \%\}$ & $+0,23 \%$ & $4,77 \%\}$ \\
\hline $\begin{array}{l}+0,19 \% \\
+0,09 \%\end{array}$ & $\left.\begin{array}{l}9,26 \% \\
8,50 \%\end{array}\right\}$ & & \\
\hline
\end{tabular}
weichen flüchtiger Verbindungen während der Eindampfung in der Tat immer statthat, und zwar auch dann, wenn wir die Trockensubstanz durch überwiegende Wasseraufnahme vermehrt finden, geht auch aus nachfolgender Zusammenstellung hervor: 
Wir sehen hier zwar die Trockensubstanz vom anfänglichen Gewichtsverlust $\mathrm{zu}$ wachsender Gewichtszunahme übergehen; wir sehen aber auch, dass von den jeweiligen beiden Parallelversuchen derjenige einen stärkeren Gewichtsverlust resp. eine geringere $\mathrm{Zu}$ nahme der Trockensubstanz aufweist (fett gedruckt), in welchem der Wassergehalt des eingedampften Rückstandes geringer (fett gedruckt) war, wo also der Eintrocknungsprozess stärker ausgefallen war. (Diejenigen Ziffern, die dieser Regel nicht entsprechen, sind in Klammern gesetzt.)

Wir können also sagen, dass die Hydrolyse des Eiweisspulvers durch Eindampfen mit destilliertem Wasser mit intramolekularer Wasseraufnahme, daher auch mit Gewichtszunahme seiner Trockensubstanz verbunden ist; diese Hydrolyse schreitet bei Wiederholung des Eindampfungsprozesses jedesmal wejter, nur wird die Gewichtszunahme durch gleichzeitigen Substanzverlust mehr oder minder beeinträchtigt.

Was den Energiegehalt des mit destilliertem Wasser eingedampften Eiweisspulvers anbelangt, so sehen wir denselben nicht unerheblich zunehmen. Dass dieser Energiezuwachs nicht vom Energiegehalt des zugesetzten und etwa nicht vollständig verflüchtigten Toluols herrührt, geht daraus hervor, dass ich bei einer Anzahl von Parallelversuchen in einem derselben (in der Tabelle mit Tol bezeichnet) Toluol, in anderen aber keines verwendete und nirgends eine regelmässige Koinzidenz zwischen Toluolzusatz und Energiezuwachs sah.

Während die Trockensubstanz, wie erwähnt, bei Wiederholung der Eindampfung jedesmal eine weitere Vermehrung erfährt, hängt die Grösse des Energiezuwachses bloss von dem Grade der Eintrocknung $a b$, d. h. sie fällt um so geringer aus, je stärker die zur kalometrischen Verbrennung notwendige Eintrocknung des Gemisches ausgefallen ist und umgekehrt.

Es ist dies nachfolgender Zusammenstellung, in welcher die Daten nach abnehmendem Wassergehalt des eingetrockneten Rückstandes geordnet sind, gut zu entnehmen, wenn auch einige Ziffern dieser Regel nicht genau folgen. 


\begin{tabular}{cc|cc}
$\begin{array}{c}\text { Energie- } \\
\text { zuwachs }\end{array}$ & $\begin{array}{c}\text { Wassergehalt des } \\
\text { eingetrockneten } \\
\text { Rückstandes }\end{array}$ & $\begin{array}{c}\text { Energie- } \\
\text { zuwachs }\end{array}$ & $\begin{array}{c}\text { Wassergehalt des } \\
\text { eingetrockneten } \\
\text { Rückstandes }\end{array}$ \\
$+1,49 \%$ & $9,26 \%$ & $+0,29 \%$ & $5,83 \%$ \\
$+1,09 \%$ & $8,50 \%$ & $+0,55 \%$ & $5,57 \%$ \\
$+0,51 \%$ & $7,78 \%$ & $+0,60 \%$ & $5,36 \%$ \\
$+0,41 \%$ & $7,11 \%$ & $-0,05 \%$ & $5,08 \%$ \\
$+0,49 \%$ & $6,03 \%$ & $+0,22 \%$ & $4,77 \%$
\end{tabular}

Dieses Verhalten des Energiezuwachses kann zweierlei Ursachen haben:

1. Es könnte sein, dass während der Eindampfung chemische Verbindungen auf endothermale Weise entstehen, die bis zu einem gewissen Eintrocknungsgrade sich erhalten können, bei stärkerem Eintrocknen hingegen wieder, und zwar exothermal zerfallen. Wie weiter unten (S. 476) gezeigt werden soll, hat diese Erklärung der experimentellen Prüfung nicht standgehalten.

2. Oder es kann von dem Grade der Eintrocknung abhängen, ob energiehaltige flüchtige Verbindungen in grösserer oder geringerer Menge entweichen und hierdurch dem Energiezuwachs einen grösseren oder kleineren Abbruch tun. Dass diese Erklärung die richtige ist, und dass während des Eindampfens ein Substanzverlust statthat, haben wir bei der Beschreibung des Verbaltens der Trockensubstanz gesehen.

Der Umstand nun, dass der Energiezuwachs - im Gegensatz zur Trockensubstanzvermehrung - mit Wiederbolung der Eindampfung keine Steigerung zeigt, kann entweder darin begründet sein, dass beim ersten Eindampfen gewisse endothermale Vorgänge sich abspielen, die bei wiederholter Eindampfung ihrerseits sich nicht wiederholen, oder aber auch davon herrühren, dass das Gewicht der erwähnten flüchtigen Substanzen zwar geringer ist, als dass es die durch Wasseraufnahme bedingte Zunahme der Trockensubstanz verdecken könnte, ihr Energiegebalt aber so hoch ist, dass durch ihren Abgang eine fortschreitende, wenn auch geringe Zunahme des Energiezuwachses vollständig maskiert wird. Freilich müsste dann für Versuch $I \mathrm{a}$ und $\mathrm{b}$ angenommen werden, dass unter gewissen Eindampfungsbedingungen bei einer ersten Eindampfung flüchtige Substanzen von geringem Energiegehalt in grossen Mengen entweichen können, daher wohl einen Verlust un Trockengehalt bedingen, jedoch keine wesentliche Schmälerung des Energiezuwachses bedingen können. 
Wenn auch es also dahingestellt bleiben muss, ob bei Wiederholung der Eindampfung eine zunehmende Energieanhäufung statthat oder nicht, kann immerhin mit Sicherheit gesagt werden, dass

1. der Energiegehalt des Eiweisspulvers durch Eindampfen mit destilliertem Wasser eine merkliche Zunahme erfährt,

2. dass der Trockengehalt des auf diese Weise behandelten Eiweisspulvers durch intramolekulare Wasseraufnahme im grossen und ganzen eine um so stärkere Zunahme erfährt, je öfter die Eindampfung wiederholt wird:

\section{B. Einwirkung der SaIzsäure.}

Wie erwähnt, wurde die Salzsäure, die versehieden lange Zeit auf Eiweiss eingewirkt hatte, vor dem Eindampfen des Gemisches durch Zugabe einer äquivalenten Menge von Natronlauge genau neutralisiert; das Eindampfen des Eiweisses resp. seiner Umwandlungsprodukte erfolgte daher eigentlich in einer Kochsalzlösung von zunehmender Konzentration. Da - wie wir gesehen haben - bereits das Eindampfen mit destilliertem Wasser eine bemerkenswerte Veränderung im Trockengehalt und im Energiegehalt des Eiweisses erzeugen kann, musste - um die Einwirkung der Salzsäure richtig beurteilen zu können - zunächst festgestellt werden, von welchen Folgen das Eindampfen in der starken Salzlösung begleitet sei. Es wurden daher beim Ansetzen der Gemische zwei Portionen sofort wieder neutralisiert und eingedampft. (Versuche $I a$ und $b$ auf Tab. IV). Wie ersichtlich, hat dieses Eindampfen eine recht bedeutende Zunahme der Trockensubstanz $(0,89-0,98 \%)$ und auch des Energiegehaltes $(1,89-2,49 \%)$ zur Folge. Die Zunahme ist weit grösser als bei dem Eindampfen mit destilliertem Wasser. Nun besteht aber insofern kein prinzipieller Unterschied zwischen den beiden Versuchsarten, als ja bei dem „Eindampfen mit destilliertem Wasser" ein grosser Teil der 6,66\% betragenden Asche des Eiweisspulvers in Iösung geht, mithin es sich auch hier um. Eindampfen mit einer, wenn auch weniger konzentrierten Salzlösung handelt.

Die Verschiedenheit in dem Erfolg der beiden Prozeduren kann

1. entweder darin liegen, dass bei stärkerer Konzentration der Salze eine stärkere Hydrolyse erfolgt, 
2. oder aber darin, dass während der kurzen Zeit (wenige Minuten), die bis zum Neutralisieren der Säure vergeht, ein anderer endothermaler Vorgang sich abspielt, der in dem bedeutenderem Energiezuwachs sich wiederspiegelt.

Um diese Verhältnisse zu klären, habe ich in Versuch II a und $b$ Säure und Lauge vorher vermischt und erst die nun entstandene Kochsalzlösung dem Eiweisspulver zugezetzt und dann eingedampft. Das Resultat war im grossen und ganzen dasselbe wie in den Versuchen $I$ a und $b$, wenn auch die Zunahme von Trockensubstanz und Energiegehalt etwas geringer ausfiel.

Es muss also von der stärkeren Konzentration der Salze verursacht sein, wenn Eiweisspulver, mit vorher neutralisierter Salzsäure eingedampft, eine bedeutendere Zunahme seines Trockengehaltes und seines Energiegehaltes erfahren, als wenn das Eindampfen mit destilliertem Wasser allein erfolgt.

Von einer Säurewirkung werden wir demnach in den Versuchen III, IV und V, wo die Säure 2, 4 und 6 Tage lang auf das Eiweiss einwirken konnte, nur dann sprechen können, wenn die Zunahme von Trockengehalt und Energiegehalt als grösser sich erweisen, als in den soeben besprochenen Versuchen I und II, wo eine Säurewirkung fast (I) oder ganz (II) auszuschliessen ist.

Wie den Daten in Tab. IV zu entnehmen ist, sind diese Zunahmen in der Tat wesentlich grösser: $1,45-3,64 \%$ bei dem Trockengehalt, $1,74-3,35 \%$ bei dem Energiegehalt. -- Eine Progression mit der Dauer der Einwirkung lässt sich bezüglich der Trockensubstanz in den Versuchen III und IV ohne weiteres konstatieren. Weniger deutlich ist sie bezüglich des Energiegehaltes, der von dem Wassergehalt des eingedampften Rückstandes (wie auf S. 472) stark beeinflusst wird. In den Versuchen II bis IV stehen dem kleinsten Wassergehalt $(4,32 \%)$ anch der kleinste Energiezuwachs $(1,74 \%)$, dem grössten Wassergehalt $(5,33 \%)$ der grösste Energiezuwachs $(3,35 \%)$ gegenüber.

Auch hier bestände also die Möglichkeit, die (S. 472) bei Besprechung der Eindampfung mit destilliertem Wasser erwähnt war, dass wäbrend des Eindampfens chemische Verbindungen auf endothermale Weise entstehen, bei stärkerer Eintrocknung jedoch wieder exothermal zerfallen. 
Untersuchung über die Wärmetönung von Enzymreaktionen IV.

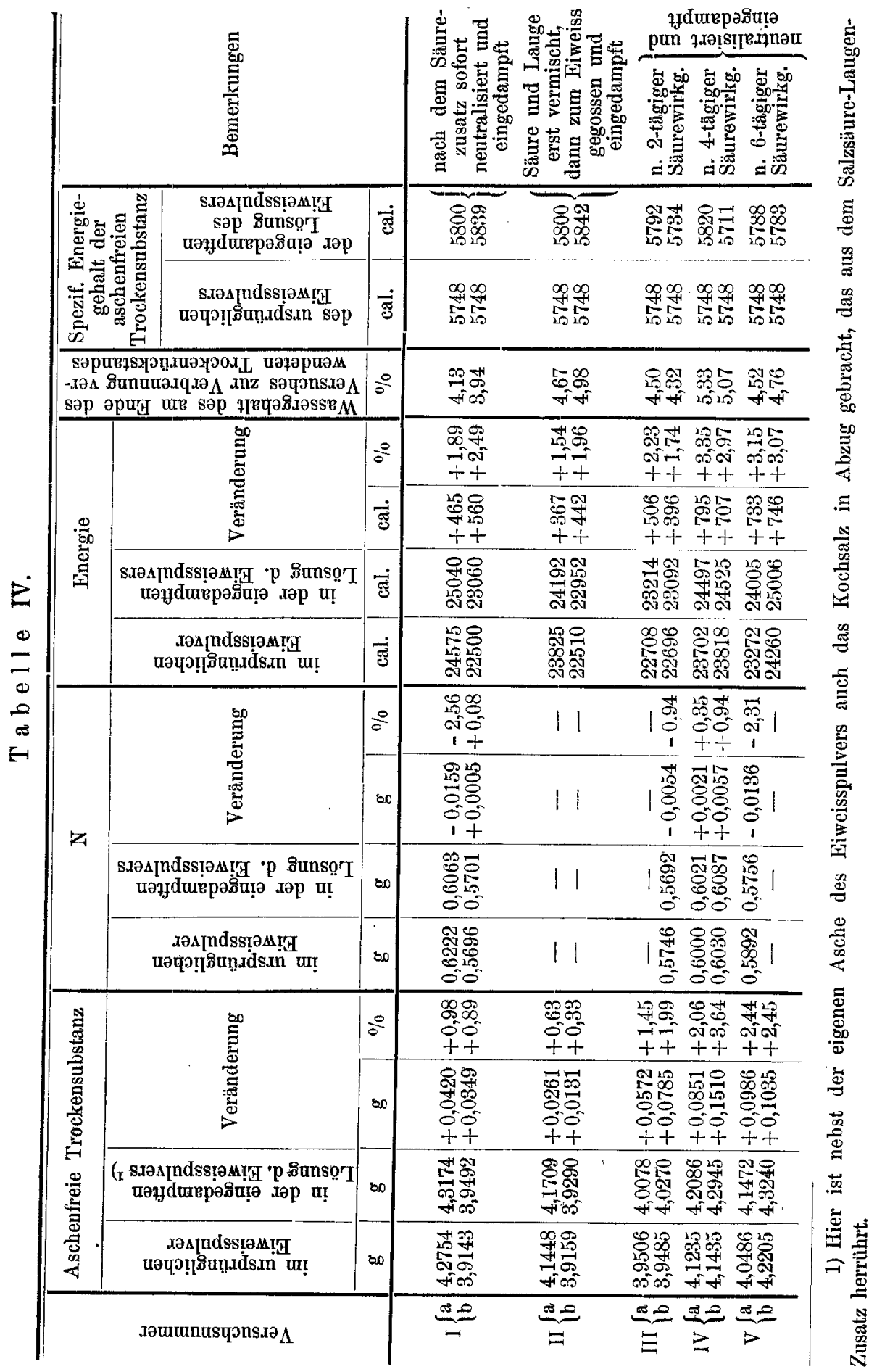


Wenn dies der Fall ist, müsste ich aus der kalometrischen Verbrennung der Pastillen verschiedene Werte für den Energiegehalt des gesammten Gemisches berechnen können, je nach dem die Pastille in lufttrockenem Zustande, also mit dem ursprünglichen Wassergehalt, oder aber nach vorangehendem Trocknen bei höherer Temperatur bis zur Gewichtskonstanz verbrannt wurde.

Ich habe also in den Versuchen I a, II b

1. einen Teil der Pastillen in $\mathrm{d}$ e $\mathrm{m}$ Zustande, d. h. mit $\mathrm{dem}$ Wassergehalt kalometrisch verbrannt, wie ich sie aus dem lufttrockenen Rückstande gepresst hatte:

Verbrennungswärme der lufttrockenen Pastille dividiert durch deren Gewicht und multipliziert mit dem Gewicht des lufttrockenen Rückstandes ${ }^{1}$ ) ergab dann den Energiegehalt des gesamten Gemisches. (Siehe Kolonne 11 der Tabelle IV).

2. Ein anderer Teil der Pastillen wurde bei $90^{\circ}$ im Vakuum bis zur Gewichtskonstanz getrocknet und nun kalometrisch verbrannt. Wenn das oben erwähnte exothermale Zerfallen wahr ist, so muss:

Verbrennungswärme der vakuumgetrockneten Pastille dividiert durch deren Gewicht und multipliziert mit dem Gewicht der Trockensubstanz des Rückstandes ${ }^{1}$ ) einen geringeren, und zwar um den ganzen Energiezuwachs geringeren Wert ergeben, als aus der Verbrennung der lufttrockenen Pastille sich ergab.

Wie aus nachstehender Zusammenstellung ersichtlich ist, batte das vollständige Austrocknen keine Verminderung des Energiegehaltes zur Folge.

\begin{tabular}{|c|c|c|}
\hline \multirow[t]{2}{*}{$\begin{array}{l}\text { Versuchs- } \\
\text { nummer }\end{array}$} & \multicolumn{2}{|c|}{$\begin{array}{c}\text { Energiegehalt des Verdauungsgemisches berechnet aus der } \\
\text { Verbrennung der }\end{array}$} \\
\hline & Iufttrockenen Pastille & im Vakuum getrockn. Pastille \\
\hline I a & $\frac{3517}{0,8863} \times 6,3088=25040$ cal & $\frac{3368}{0,8142} \times 6,0482=25017 \mathrm{cal}$ \\
\hline b & $\frac{3791}{0,9788} \times 5,9261=22952 \mathrm{cal}$ & $\frac{3242}{0,7943} \times 5,6810=22987 \mathrm{cal}$ \\
\hline
\end{tabular}

Aus der im Vakuum getrockneten Pastille liess sich also einmal um 23 cal. $=0,09 \%$ weniger, ein zweites Mal um 35 Cal. $=0,15 \%$ mehr berechnen, was kaum so viel ist, als der zulässige Versuchsfehler beträgt.

1) Inklusive Asche plus zugefügtes Kochsalz. 
Da die Abhängigkeit des Energiezuwachses vom Wassergehalt des eingetrockneten Rückstandes demnach $\mathrm{n}$ i $\mathrm{ch}$ t von einem Zerfallen endothermal entstandener Verbindungen herrührt, muss hier (wie auch S. 472) angenommen werden, dass während des Eindampfens energiehaltige Verbindungen in umso grösserer Menge entweichen, je stärker eingetrocknet wurde.

Das Ergebnis einer zwei- bis sechstägigen Einwirkung von Salzsäure auf Eiereiweiss besteht nach alledem in einer intensiven Zunahme der Trockensubstanz wie auch des Energiegehaltes des Verd a u ugs gemisches. Diese Zunahme ist weit grösser als diejenige, welche das Eindampfen mit destilliertem Wasser allein erzeugt, aber auch grösser als die, welche dureh Eindampfen mit der neutralisierten Salzsäure nıt einer konzentrierten Kochsalzlösung verursacht wird.

Eine mit der Dauer der Säurewirkung parallele Progression dieser Zunahmen ist nur bezüglich des Trockengehaltes nachzuweisen.

\section{Pepsinwirkung.}

Wie auf Seite 464 ausführlich erörtert war, liessen die Ergebnisse der ersten und zweiten Versuchsreihe vermuten, dass Säurewirkung und Pepsinwirkung chemische Prozesse darstellen, deren Wärmetönungen verschieden und vielleicht gar entgegengesetzter Richtung sind, und dass die durch Säurewirkung hervorgerufene Energieanhäufung infolge der Pepsinwirkung verloren gehe, wenn nur hinreichend lang verdaut war.

Da die Unsicherheit jener Ergebnisse offenbar von dem zu geringen Pepsingehalt der Verdauungsgemische verursacht war, hatte ich in der fünften Versuchsreihe für reichlichen Pepsingehalt gesorgt, und hierdurch einerseits eine so kräftige Pepsinverdauung erreicht, dass nach zweitägiger Verdauung keine Spur mehr von koagulablem Eiweiss nachzuweisen war, andererseits aber Klarheit bezüglich der erwähnten Vermutung bekommen.

Es war nämlich nicht nur der erwartete Abfall des Energiezuwachses, sondern durchgehends in allen verdauten Gemischen ein bedeutender Energie v e r 1 ust zu konstatieren. Dieser Energieverlust kann: 
Paul Hári:

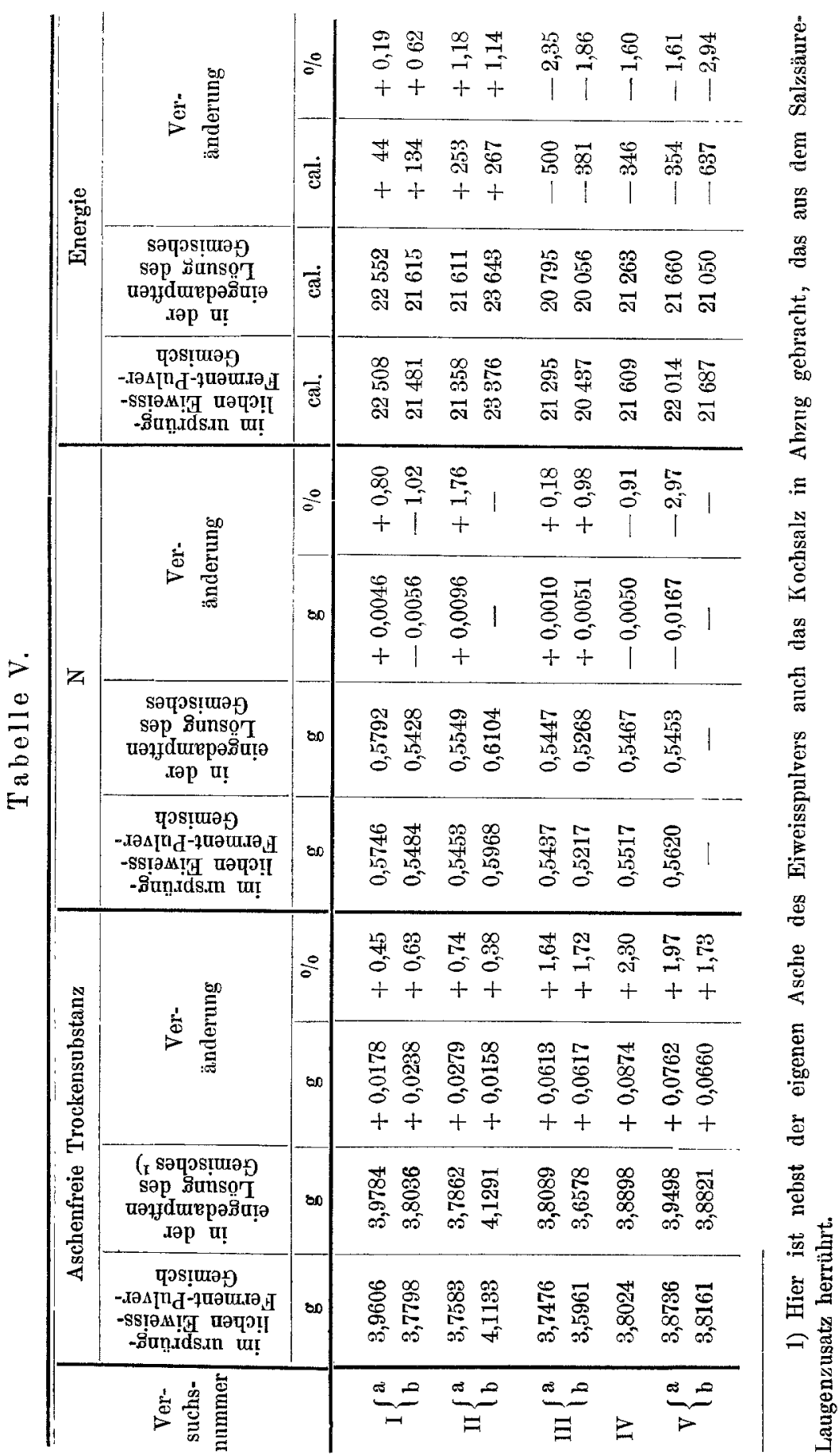


Untersuchungen über die Wärmetönung von Enzymreaktionen. IV. 479

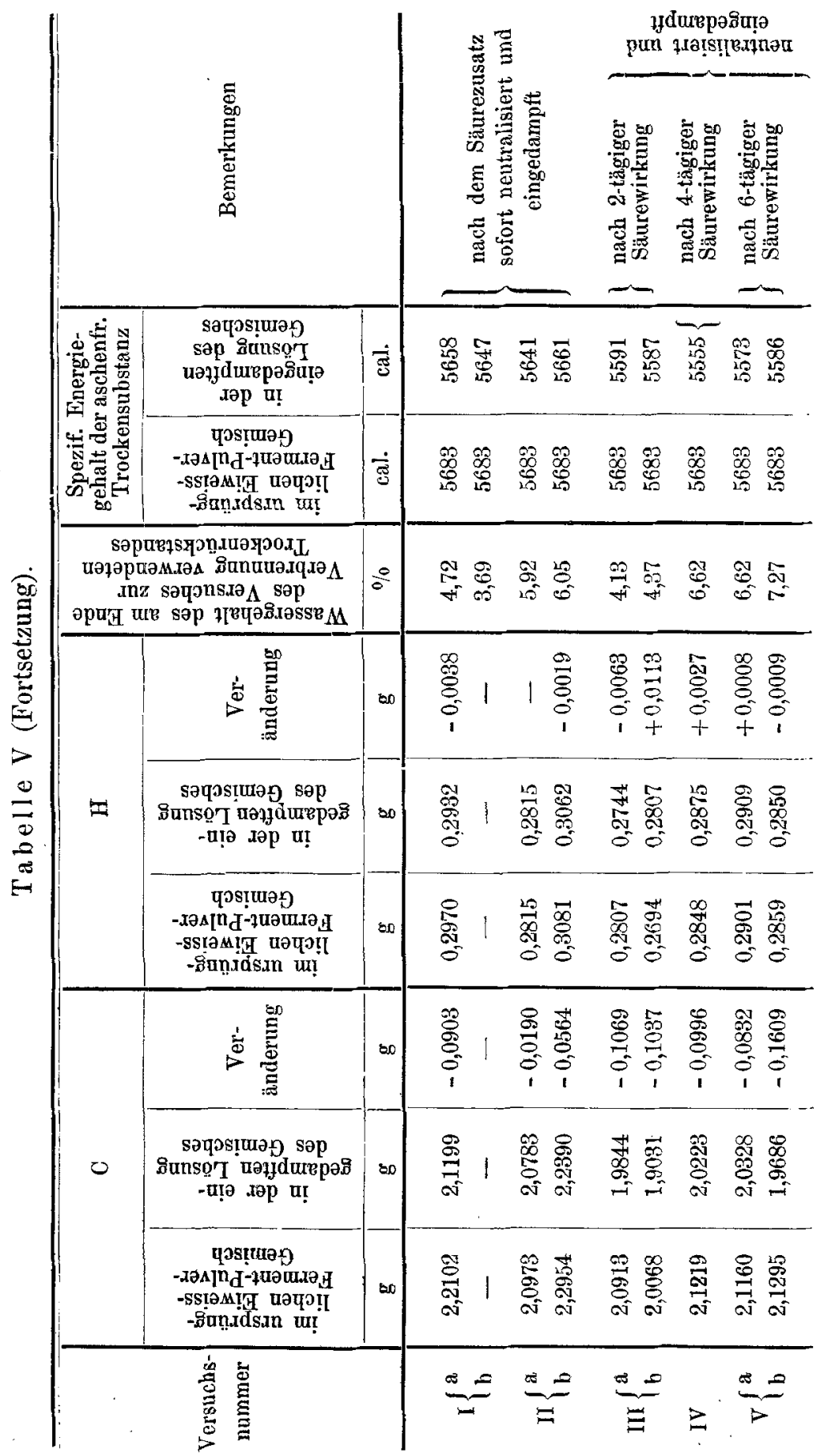


a) entweder dadurch entstanden sein, dass während der Verdauung oder während des Eindampfens energiehaltige Verbindungen sich verflüchtigen,

b) oder er kann durch einen chemischen Prozess mit positiver Wärmetönung bedingt sein,

c) oder aber auf beiderlei Arten entstehen.

Dass während der Verdauung resp. während des Eindampfens organische Substanzen sich verflüchtigen, geht aus den Daten der Elementaranalyse hervor, die ich von allen Gemischen dieser Versuchsreihe angestellt habe, und die in allen Versuchen auf ein C-Defizit des eingedampften Gemisches hinweisen.

Nun fragt es sich, ob dieser Verlust an organischer Substanz allein geeignet ist, den Energieverlust zu erklären oder nicht? Die Antwort erhalten wir aus Tabelle VI, wo die korrespondierenden Daten der 4. (Salzsäure) - und der 5. (Salzsäure-Pepsin) - Versuchsreihe nebeneinander gestellt sind. Es ist denselben zu entnehmen, dass in den Versuchen Ia, II a und II b der 4 . Versuchsreihe ein durchschnittlicher Energiezuwachs von 412 cal. erfolot, während in den entsprechenden Versuchen der 5. Versuchsreihe, in denen ein $\mathrm{C}$-Verlust $^{1}$ ) von 9 resp. 2 resp. $5 \mathrm{eg}$ festgestellt wurde, jener Zuwachs um 381 resp. 172 resp. 178 cal. geschmälert erscheint $^{2}$ ), wobei aber immer ein geringer Energiezuwachs übrig bleibt.

Dem gegenüber sehen wir in den vorher verdauten Gemischen IIIa; IIIb, IV und Va C-Verlusten von abermals zirka 9-10 cg

1) Der Einfachheit halber spreche ich hier immer nur von C-Verlust, während es sich ja eigentlich nicht bloss um den $\mathrm{C}$, sondern um die ganze entwichene organische Substanz handelt, in der auch $\mathrm{H}$, vielleicht auch $\mathrm{N}$ enthalten waren. Die Unterschiede im H-Gehalt sind kaum grösser, als dem zulässigen Versuchsfehler entspricht; da bei der Hydrolyse $H$ aufgenommen wird, ist es auch schwer, fast unmöglich, die Grösse des H-Verlustes festzustellen. N-Verlust jst nicht mit Sicherheit festzustellen, da die N-Werte mehrfache Unsicherheit zeigen.

2) Laut den Ausführungen auf S. 472 über den Einfluss des Eintrocknungsgrades unterliegt es keinem Zweifel, dass die Eindampfung auch in der 4. Versuchsreihe mit Verlusten an organischer, energiehaltiger Substanz einhergeht; anstatt die ganzen 2 resp. 5 resp. $9 \mathrm{cg}$ Kohlenstoff für den Energieabfall verantwortlich zu machen, wäre es demnach richtiger, hier nur den Mehrverlust (im Vergleiche zur 4. Versuchsreihe) in Betracht zu ziehen. Da aber in der 4. Versuchsreihe eine C-Bëstimmung aus äusseren Gründen nicht vorgenommen werden konnte, muss dieser Fehler wohl oder übel mit in den Kauf genommen werden. 
nicht bloss einem Abfall des Energiezuwachses, sondern direktem Energieverlust von $350-500$ cal. entsprechen.

Tabelle VI.

\begin{tabular}{|c|c|c|c|c|c|c|}
\hline \multirow{2}{*}{$\begin{array}{l}\text { Versuchs- } \\
\text { nummer }\end{array}$} & \multicolumn{2}{|c|}{$\begin{array}{l}\text { Zunahme der } \\
\text { Trockensubstanz }\end{array}$} & \multicolumn{2}{|c|}{$\begin{array}{l}\text { Veränderung des } \\
\text { Energiegehaltes }\end{array}$} & \multirow{2}{*}{\begin{tabular}{|c|}
$\begin{array}{c}\text { C-Defizit der } \\
\text { ein- } \\
\text { gedampften } \\
\text { Gemische }\end{array}$ \\
5. \\
$\begin{array}{c}\text { Versuchs- } \\
\text { reihe } \\
\mathrm{g}\end{array}$ \\
\end{tabular}} & \multirow{2}{*}{$\begin{array}{l}\text { Be- } \\
\text { merkungen }\end{array}$} \\
\hline & $\begin{array}{c}4 . \\
\text { Versuchs- } \\
\text { reihe } \\
\mathrm{g}\end{array}$ & $\begin{array}{c}5 . \\
\text { Versuchs- } \\
\text { reihe } \\
\mathrm{g}\end{array}$ & $\begin{array}{c}4 . \\
\text { Versuchs- } \\
\text { reihe } \\
\text { cal. }\end{array}$ & $\begin{array}{c}5 . \\
\text { Versuchs- } \\
\text { reihe } \\
\text { cal. }\end{array}$ & & \\
\hline$I\left\{\begin{array}{l}a \\
b\end{array}\right.$ & $\begin{array}{l}0,0420 \\
0,0349\end{array}$ & $\begin{array}{l}0,0178 \\
0,0238\end{array}$ & $\begin{array}{l}+465 \\
+560\end{array}$ & $\begin{array}{l}+\quad 44 \\
+134\end{array}$ & $\begin{array}{c}0,0903 \\
-\end{array}$ & ohne \\
\hline II $\left\{\begin{array}{l}a \\
b\end{array}\right.$ & $\begin{array}{l}0,0261 \\
0,0131\end{array}$ & $\begin{array}{l}0,0279 \\
0,0158\end{array}$ & $\begin{array}{l}+367 \\
+442\end{array}$ & $\begin{array}{l}+253 \\
+267\end{array}$ & $\begin{array}{l}0,0190 \\
0,0564\end{array}$ & eingedampft \\
\hline $\operatorname{III}\left\{\begin{array}{l}a \\
b\end{array}\right.$ & $\begin{array}{l}0,0572 \\
0,0785\end{array}$ & $\begin{array}{l}0,0613 \\
0,0617\end{array}$ & $\begin{array}{l}+506 \\
+396\end{array}$ & $\begin{array}{l}-500 \\
-381\end{array}$ & $\begin{array}{l}0,1069 \\
0,1037\end{array}$ & \\
\hline $\operatorname{IV}\left\{\begin{array}{l}a \\
b\end{array}\right.$ & $\begin{array}{l}0,0851 \\
0,1510\end{array}$ & $\underline{0,0874}$ & $\begin{array}{l}+795 \\
+707\end{array}$ & -346 & $\stackrel{0,0996}{-}$ & $\begin{array}{c}\text { vorher } \\
\text { verdaut, dann } \\
\text { eingedampft }\end{array}$ \\
\hline $\mathrm{V}\left\{\begin{array}{l}\mathrm{a} \\
\mathrm{b}\end{array}\right.$ & $\begin{array}{l}0,0986 \\
0,1085\end{array}$ & $\begin{array}{l}0,0762 \\
0,0660\end{array}$ & $\begin{array}{l}+733 \\
+746\end{array}$ & $\begin{array}{l}-354 \\
-637\end{array}$ & $\begin{array}{l}0,0832 \\
0,1609\end{array}$ & \\
\hline
\end{tabular}

Wenn je ne $9 \mathrm{eg}$ (der nicht verdauten Gemische) bloss einen Abfall des Energiezuwachses im Werte von 400 cal. verursachen, können doch diese $9-10 \mathrm{cg}$ (der verdauten Gemische) nicht einen Verlust von $350-500$ cal., das ist einen Abfall von $750-900$ cal. rechtfertigen? ${ }^{1}$ )

An diesem Energieverlust muss demnach - nebst dem Entweichen organischer flüchtiger Verbindungen - noch ein anderer Faktor beteiligt sein! Dass dem auch so ist, geht am besten aus einem Vergleich der auf Versuch III a, III b und IV a der beiden Versuchsreihen bezüglichen Daten hervor. Da hier die Zunahme der Trockensubstanz in beiden Versuchsreihen annähernd dieselbe ist, so kann füglich angenommen werden, dass auch die C-Verluste, die bloss in der 5. Versuchsreihe bestimmt wurden, in der 4. Versuchsreihe gleich gross waren. Trotzdem sehen wir hier eine Zunahme des Energiegehaltes um 500-800 cal., dort aber einen Verlust von $350-500$ cal., also einen Gesamtunterschied von $850-1300$ cal.

1) Man müsste denn annehmen, dass aus den verdauten Gemischen Substanzen bedeutend höherer Energiegehaltes entweichen. 
Dies kann selbstverständlich kein Zufall sein! Allerdings ist es möglich, dass in den verdauten Gemischen die Pepsin-SalzsäureReihe organische Verbindungen von höherem Energiegehalt sich verflüchtigen als in den vorher nicht verdauten Gemischen dieser Reihe resp. als in sämtlichen Gemischen der 4. Versuchsreihe, die bloss Salzsäure, jedoch kein Pepsin enthielten. Dass aber korrespondierende Gemische beider Reihen an Menge wohl annähernd gleiche, an Energiegehalt aber, also auch chemisch völlig verschiedene Verbindungen verlören, kann man wohl kaum annehmen.

So lässt sich wohl mit Recht aussprechen, dass die PepsinSalzsäure-Wirkung im Gegensatzzur reinen Salzsäurewirkung mit positiver Wärmetönung einhergeht, wodurches nicht nur zu einem Abfall der durch Salzsäure bewirkten Energieanhäufung, sondern auchzu einem wirklichen Verlust an chemischer Energie kommt.

Es ergibt sich nun die weitere Frage, ob dieser Energieverlust mit dem Fortschreiten der Verdauung ebenfalls zunehme oder aber sich ebensowenig verändere, wie dies bezüglich der durch Säure bewirkten Zunahme des Energiegehaltes in der vierten Versuchsreihe (S. 474) der Fall ist?

Bei der Unmöglichkeit, die kalometrische Bestimmung ohne Eindampfung bei höherer Temperatur ${ }^{1}$ ), also ohne Substanzverlust zu bewerkstelligen, sind die auf Energieverluste bezüglichen Werte immer mit ansehnlichen Fehlern behaftet. Soweit aber den Daten der 5. Versuchsreihe zu entnehmen ist, scheint es, dass die Energieverluste (mit Ausnahme des Versuches Vb) von der Dauer der Verdauung nicht abhängen, es sich also um einen einmaligen definitiven Energieverlust handeln dürfte, wie auch der durch Săure bewirkte Energiezuwachs ein einmaliger (und nicht progressiver) $\mathrm{zu}$ sein scheint.

1) Siehe auch meine Trypsinarbeit, 1. c. 